\title{
Activation and potentiation of GIRK channel activity by ivermectin
}

\author{
I-Shan Chen ${ }^{1,3}$, Michihiro Tateyama ${ }^{1,3}$, Yuko Fukata ${ }^{2,3}$, Motonari Uesugi, $^{4,5}$, Yoshihiro Kubo ${ }^{1,3}$
}

\begin{abstract}
${ }^{I}$ Division of Biophysics and Neurobiology, Department of Molecular and Cellular Physiology, National Institute for Physiological Sciences, Japan, ${ }^{2}$ Division of Membrane Physiology, Department of Molecular and Cellular Physiology, National Institute for Physiological Sciences, Japan, ${ }^{3}$ Department of Physiological Sciences, School of Life Science, SOKENDAI (The Graduate University for Advanced Studies), Japan, ${ }^{4}$ Institute for Integrated Cell-Material Sciences (WPI-iCeMS), Kyoto University, Japan, ${ }^{5}$ Institute for Chemical Research, Kyoto University, Japan
\end{abstract}

G-protein-gated inwardly rectifying $\mathrm{K}^{+}$(GIRK) channels control various physiological functions. For example, GIRK1/2 heterotetramers in the brain regulate neuronal excitability; GIRK1/4 heterotetramers in the heart regulate heart rate. Recently, we identified a novel GIRK activator, ivermectin (IVM), which is a widely used antiparasitic drug in humans and pets that activates glutamate-gated $\mathrm{Cl}^{-}(\mathrm{GluCl})$ channel in parasites. It is known that IVM binds to the transmembrane domains (TMs) of several ligand-gated channels, such as Cys-loop receptors and P2X receptors. In the present study, we showed the effects and regulation mechanisms of GIRK channel by IVM. Electrophysiological recordings in Xenopus oocytes revealed that IVM activates GIRK2 more efficiently than GIRK4. We found that IVM activates GIRK channel in a phosphatidylinositol-4,5-biphosphate ( $\left.\mathrm{PIP}_{2}\right)$-dependent manner, and that the IVM-mediated GIRK activation is independent of $\mathrm{G}_{\beta \gamma}$ subunits. We also observed that IVM not only acts as an activator but also as a modulator of GIRK channel that potentiates the M2 muscarinic receptor-mediated GIRK activation. Chimeric and mutagenesis analyses identified an amino acid residue unique to GIRK2 among the GIRK family, Ile82, located in the slide helix between the TM1 and the N-terminal cytoplasmic tail domain (CTD), which is critical for the activation. The results demonstrate that the TM-CTD interface in GIRK channels, rather than the TMs, governs IVM-mediated activation. These findings provide us with novel insights on the mode of action of IVM in ion channels that could lead to identification of new pharmacophores which activate the GIRK channel. 